\title{
The Kaiser Permanente Northern California research program on genes, environment, and health (RPGEH) pregnancy cohort: study design, methodology and baseline characteristics
}

\author{
M. M. Hedderson", A. Ferrara, L. A. Avalos, S. K. Van den Eeden, E. P. Gunderson, D. K. Li, A. Altschuler, S. Woo, \\ S. Rowell, V. Choudhary, F. Xu, T. Flanagan, C. Schaefer and L. A. Croen
}

\begin{abstract}
Background: Exposures during the prenatal period may have lasting effects on maternal and child health outcomes. To better understand the effects of the in utero environment on children's short- and long-term health, large representative pregnancy cohorts with comprehensive information on a broad range of environmental influences (including biological and behavioral) and the ability to link to prenatal, child and maternal health outcomes are needed. The Research Program on Genes, Environment and Health (RPGEH) pregnancy cohort at Kaiser Permanente Northern California (KPNC) was established to create a resource for conducting research to better understand factors influencing women's and children's health. Recruitment is integrated into routine clinical prenatal care at KPNC, an integrated health care delivery system. We detail the study design, data collection, and methodologies for establishing this cohort. We also describe the baseline characteristics and the cohort's representativeness of the underlying pregnant population in KPNC.

Methods: While recruitment is ongoing, as of October 2014, the RPGEH pregnancy cohort included 16,977 pregnancies (53\% from racial and ethnic minorities). RPGEH pregnancy cohort participants consented to have blood samples obtained in the first trimester (mean gestational age 9.1 weeks $\pm 4.2 \mathrm{SD}$ ) and second trimester (mean gestational age 18.1 weeks $\pm 5.5 \mathrm{SD}$ ) to be stored for future use. Women were invited to complete a questionnaire on health history and lifestyle. Information on women's clinical and health assessments before, during and after pregnancy and women and children's health outcomes are available in the health system's electronic health records, which also allows long-term follow-up.

Discussion: This large, racially- and ethnically-diverse cohort of pregnancies with prenatal biospecimens and clinical data is a valuable resource for future studies on in utero environmental exposures and maternal and child perinatal and long term health outcomes. The baseline characteristics of RPGEH Pregnancy Cohort demonstrate that it is highly representative of the underlying population living in the broader community in Northern California.
\end{abstract}

Keywords: Pregnancy, Cohort, Resource, Biorepository, Maternal health

* Correspondence: Monique.M.Hedderson@kp.org

Kaiser Permanente Northern California Division of Research, 2000 Broadway,

Oakland, CA 94612, USA 


\section{Background}

Exposures before and during pregnancy contribute to the immediate and future health outcomes of both women and their children. Emerging evidence supports the notion that the prenatal period is a critical developmental window during which in utero exposures may have lasting effects on a child's future health [1, 2]. Biological programming [3] occurs during fetal life in response to in utero exposure to nutrient substrates, hormones, growth factors, cytokines, environmental conditions or toxins, and other exposures. Evidence also shows that women who develop pregnancy complications are at increased risk of developing chronic diseases later in life [4-7]. However, the mechanisms underlying many of these findings remain unclear, and further research is needed to advance our understanding of how the in utero environment impacts the short- and long-term health of both the woman and her child.

Large studies with multiple measurements of biomarkers during pregnancy are needed to better measure perinatal exposures and to understand the etiologically relevant period of the effects of exposures on perinatal outcomes. To fully understand how the in utero environment influences the short- and long-term health of women and their children, large representative study populations with comprehensive information on a broad range of factors, including biomarkers, medical conditions, medications, nutrition, physical activity and environmental exposures, are needed.

The Kaiser Permanente Northern California (KPNC) Research Program on Genes, Environment, and Health (RPGEH) has established a large pregnancy cohort that integrates biospecimens with rich and accurate clinical and health data available from the electronic health record (EHR), creating a unique resource available to advance research on women's and children's health. The establishment of this pregnancy cohort within an integrated health care delivery system with an EHR has the additional advantage of enabling accurate assessment of short- and long-term maternal and child health outcomes and the rapid translation of clinically meaningful findings into clinical practice. This report describes the design and methods used to establish this pregnancy cohort and its biorepository in KPNC. We present preliminary data on the baseline characteristics of the cohort to demonstrate its racial-ethnic diversity and the prevalence of several perinatal complications of interest, as well as its representativeness with regard to the underlying population of pregnancies at KPNC. We further discuss possible use of this large cohort including the ability to efficiently follow it prospectively through the EHR to answer pressing questions regarding women's and children's health.

\section{Methods/Design}

Aim

The aim of this project is to establish a large pregnancy cohort that integrates biospecimens with rich and accurate clinical and health data to create a resource to advance scientific research on women's and children's health. The pregnancy cohort is able to be linked to short- and longterm maternal and child health outcomes to facilitate the rapid translation of clinically meaningful findings into clinical practice.

\section{Design}

The KPNC Division of Research started the Research Program on Genes, Environment and Health (RPGEH) in 2007 to develop a genetic epidemiology population resource which integrates data from multiple sources from consenting KPNC adult members, including biospecimens, clinical data from the EHR, lifestyle and risk factor data from surveys, and environmental exposure data from both laboratory and geographic information systems. One component of the RPGEH is the RPGEH Pregnancy Cohort.

\section{Establishment of RPGEH pregnancy cohort}

The Division of Research worked closely with KPNC clinical partners to develop facility-based recruitment procedures and laboratory blood processing workflows that could be easily integrated as part of routine prenatal medical care. The entire recruitment process was designed to become an integrated and routine part of the clinical prenatal intake process. To avoid disruption of clinical workflows, all RPGEH program-related processes (e.g., questions from patients, and follow-up) are handled by research staff. The recruitment and biospecimen collection protocol processes are described below.

\section{Study setting}

KPNC provides integrated health care to over 3.6 million members through 7,000 physicians, > 240 medical office buildings and 22 hospitals. The KPNC service area spans 14 counties of the greater Bay Area, as well as the California Central Valley from Sacramento to Fresno and includes urban and rural areas. The population is highly representative of the demographic characteristics of the entire population from this geographic area [8]. The membership is racially and socio-economically diverse. KPNC is vertically integrated such that all care is provided in a closed system and documented in an EHR. The EHR are clinical records, not claims data, and thus are robust with regard to data quality and completeness. The membership of reproductive-aged women (15-44) includes women with KP commercial insurance (varying copays, varying deductible levels), MediCal, and other California state subsidized programs. Within KPNC, 
there are 16 delivery hospitals and approximately 38,000 pregnancies each year.

\section{Recruitment of participants}

The RPGEH Pregnancy Cohort recruitment began in February 2010 at the KPNC Walnut Creek outpatient medical facility. Recruitment gradually expanded to cover almost the entire KPNC service area. Figure 1 shows the geographical locations of RPGEH pregnancy cohort members in an area of over 28,000 square miles, an area slightly larger than South Carolina. Clinical staff, such as medical assistants and nurses at the Obstetrics and Gynecology department, routinely gives a RPGEH pregnancy cohort flyer with frequently asked questions and a consent form to women at the initial prenatal visit. They also briefly describe the RPGEH Pregnancy Cohort and ask women if they would like to participate. If the woman agrees to participate and signs the consent form, the clinic staff places the research blood draw order in the woman's EHR.

\section{Biospecimen collection and storage process}

Women who consent have blood drawn for research purposes into one $8.5 \mathrm{~mL}$ serum separator tube (SST) tube and one $6.0 \mathrm{~mL}$ ethylenediaminetetraacetic Acid (EDTA) tube at the same time as the clinically ordered blood tests at her local KPNC laboratory at two times during their pregnancy: in the first trimester during a standard first trimester panel or genetic screening $(\sim 10-13$ weeks, 6 days) and during the second trimester either along with standard genetic screening ( 15-20 weeks) or with the gestational diabetes screening ( 24-28 weeks). The blood tubes are couriered as part of the normal KPNC laboratory system to the Regional Laboratory, where they are transferred to the RPGEH Biorepository (see description below) and further processing occurs.

\section{The RPGEH Research Biorepository}

The RPGEH Biorepository is a state-of-the-art research biorepository and staffed with research laboratory personnel who are responsible for maintaining the laboratory space, checking in, processing and storing samples, and retrieving aliquots for studies. Equipment includes an ABF 500 automated blood fractionation robot unit, an RTS A4 temperature and humidity controlled robotic ambient storage unit for archiving DNA using Biomatrica DNA stable storage medium, and a walk-in- $80^{\circ} \mathrm{C}$ freezer. A custom developed Laboratory Information Management System (LIMS) tracks specimens at each step and is linkable to RPGEH operations and clinical information databases.

Once at the Biorepository, serum from the SST is aliquotted into 4, $0.8 \mathrm{~mL}$ cryovials. The EDTA tube is centrifuged and plasma is aliquoted into $2,0.8 \mathrm{~mL}$ cryovials, while $1.0 \mathrm{~mL}$ of buffy coat is aspirated and placed in a cryovial. All cryovials are stored at $-80{ }^{\circ} \mathrm{C}$.

\section{Clinical data}

Information on participants in the Pregnancy Cohort is obtained from several sources of rich clinical data (resources are described below).

\section{Information obtained from the EHR during the first prenatal visit}

As part of routine prenatal care, all pregnant women complete a prenatal questionnaire during the first trimester or shortly after the pregnancy is clinically confirmed. This questionnaire includes questions on parity, gravidity, prior delivery and birth history, reproductive history, menstrual history, prior medical history, social circumstances (e.g., stress, domestic violence, etc.), and an Adult Outcomes Questionnaire (AOQ) which includes the PHQ-9 $[9,10]$ depression screener and the Generalized Anxiety Disorder scale (GAD-2) [11] as well as functioning items. The information from the Prenatal Questionnaire is recorded in the KPNC EHR for access to extensive health and reproductive history on the cohort. Several other sources of pre-pregnancy information are available in the EHR including pre-pregnancy body mass index (BMI) if a woman had been a KPNC member prior to conception.

\section{Early start substance use data}

In addition to the Prenatal Questionnaire, a selfadministered Early Start Program Prenatal Substance Use Screening Questionnaire is completed at entry into prenatal care. Early Start is an integrated prenatal program to intervene when a pregnant woman reports alcohol, tobacco and other drug use during pregnancy [12]. The questionnaire asks about substance use before pregnancy and since pregnancy began, including alcohol, smoking, and prescription drug use.

\section{Clinical data available in KPNC EHR}

KPNC maintains complete databases that capture all encounters including hospitalizations, outpatient visits, radiology/imaging, laboratory tests, and prescription medications and combines these data for presentation to clinicians as part of the EHR. Data captured in these databases include inpatient and outpatient diagnostic information, imaging reports, laboratory tests and results, pharmacy dispenses including dosages and days of supply, and surgery outcomes, among others. All vital signs including weight and height, blood pressure and physical activity are recorded in the EHR. As noted above, these data are clinical information maintained in an EHR and are not claims data, and enable the detailed examination of diagnoses and treatments before, 
Hedderson et al. BMC Pregnancy and Childbirth (2016) 16:381

Page 4 of 10

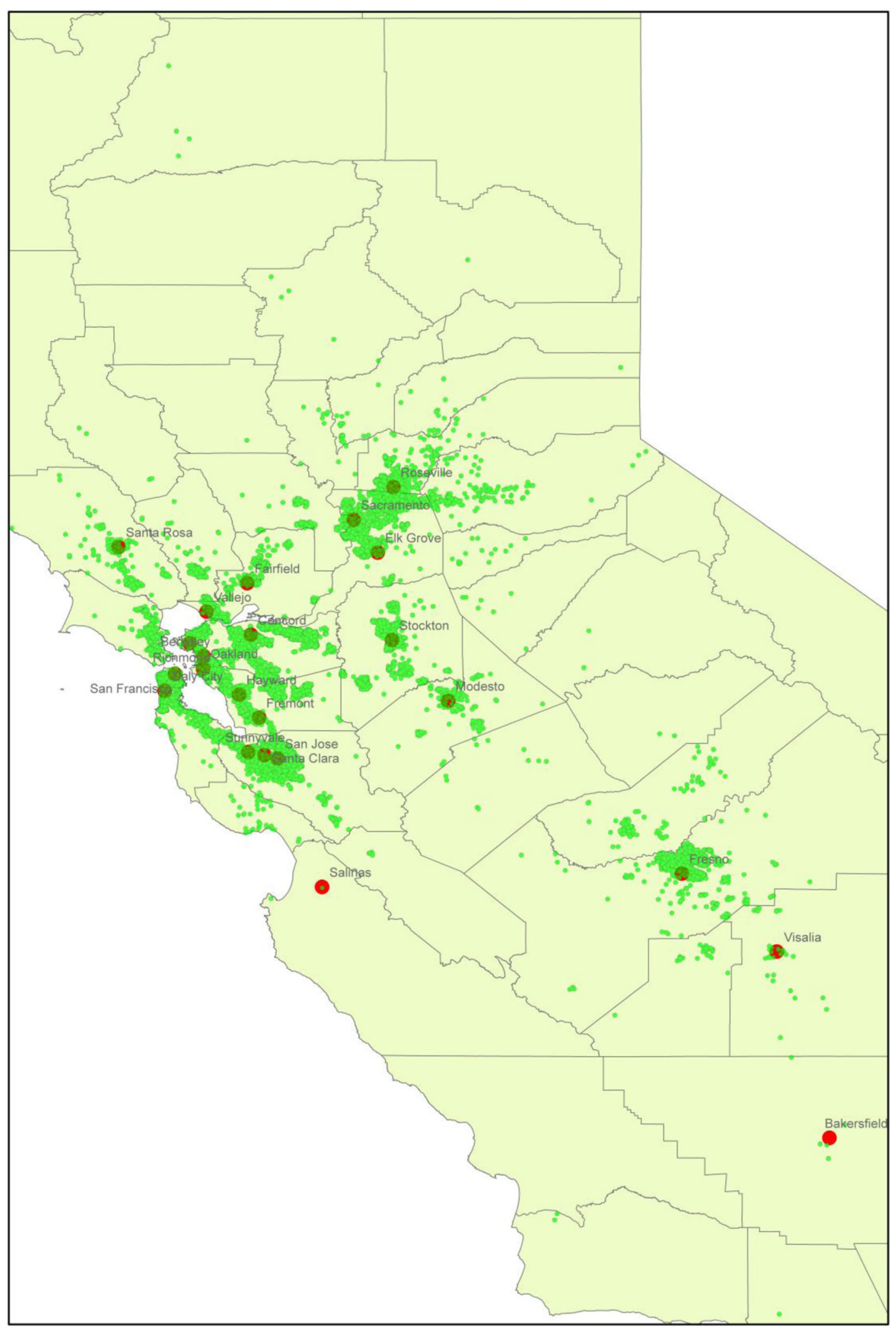

Fig. 1 Geographic locations of Kaiser Permanente Northern California RPGEH Pregnancy Cohort members 
during and after pregnancy. In addition, when an infant is born, he/she is issued a unique medical record number (MRN) that is used for all care associated with the infant. It is linkable to the mother's unique MRN that allow identification of the mother-infant pair. This allows us to also link to the women's infants and examine infant growth and outcomes at birth and during childhood, along with other health outcomes, including the mother's outcomes.

\section{RPGEH pregnancy cohort questionnaire}

To obtain more detailed information not captured in the HER, each participant is invited to complete the RPGEH Pregnancy Cohort questionnaire. The RPGEH questionnaire ascertains information about a variety of sociodemographic, lifestyle and environmental factors not routinely captured in the EHR, including diet, physical activity, multivitamin use and self-reported health history before and during the study pregnancy (Additional file 1).

\section{Environmental exposure data}

Over $98 \%$ of the RPGEH pregnancy cohort has been successfully geocoded and can be linked to contextual or environmental data, including spatiotemporal data that exist in public access databases. These data come from commercial sources, non-profit agencies, and local, regional, state and national government agencies. Data from these various sources are being incorporated into a KPNC geographic information system (GIS) database using ArcGIS software (Redlands, CA). The database will include data on retail food outlets, green space, infrastructure (roads, educational facilities, health delivery centers, and public assistance facilities), traffic density, air pollution, pesticide use, toxic sites, toxic release inventories, and other factors. Other relevant information, currently located at other agencies but available for linkage, includes water quality, centers of social congregation (e.g., religious or spiritual institutions, senior centers, youth activity centers, etc.), and crime data. California has some of the most complete publically available geospatial data across these environmental factors anywhere in the world.

Below we describe the sources used for determining the clinical outcomes of the RPGEH Pregnancy Cohort participants and non-participants for this preliminary report.

\section{Clinical outcomes during pregnancy/in utero exposure to maternal metabolism}

\section{Women's body mass index and gestational weight gain}

Through the EHR we are able to capture a woman's body mass index prior to pregnancy as well her gestational weight gain trajectory and total gestational weight gain, allowing us to assess possible determinants of gestational weight gain, as well as to define the sequelae of in utero exposure to maternal obesity and excessive gestational weight gain (i.e., over nutrition) or inadequate gestational weight gain (i.e., undernutrition) in relation to the current Institute of Medicine guidelines [13] on child health.

\section{Pregestational diabetes and gestational diabetes mellitus (GDM) and impaired glucose tolerance}

Pregestational diabetes is obtained from the KPNC Diabetes Registry [14] and GDM is obtained from the KPNC pregnancy glucose tolerance and GDM Registry [15]. These registries allow for the identification of GDM based on objective glucose measurement defined according to laboratory glucose values meeting the Carpenter and Coustan diagnostic criteria [16].

\section{Preeclampsia/Hypertensive disorder of pregnancy}

Preeclampsia and hypertensive disorders of pregnancy were also obtained from the EHR and were defined according to the following ICD-9 codes: pre-existing hypertension 642.0-642.2, gestational hypertension 642.3, preeclampsia or eclampsia 642.4-642.7. The validity of these ICD-9 codes to diagnose hypertensive disorders of pregnancy has previously been reported [17].

\section{Clinical outcomes at birth Preterm birth}

Gestational age is based on the estimated date of delivery recorded in the EHR, which is determined by the woman's self-reported last menstrual period (LMP), or by first trimester ultrasound if different from the LMP-based calculation by more than 1 week. Preterm birth was defined as birth at $<37$ weeks' gestation. We also examined the degree of preterm birth using the following definitions: extreme preterm ( $<28$ weeks' completed gestation), severe preterm (28-31 weeks' completed gestation), moderate preterm (32-33 weeks' completed gestation) and late preterm (34-36 weeks' completed gestation) [18].

\section{Infant size for gestational age}

Infant birthweight was obtained from the EHR. Large for gestational age was defined as birthweight $>90$ th percentile and small for gestational age was defined as birthweight $<10$ th percentile for the underlying KPNC population's race-ethnicity and gestational age-specific birthweight distribution [19].

\section{Cesarean delivery}

Cesarean delivery information was obtained from the KPNC neonatal and infant cohort [20] and is defined according to ICD-9 codes $654.2 \times$ for delivery mode recorded in the EHR.

Recruitment to date and prevalence of outcomes of interest Between February 2010 and October 2014, pregnant members of KPNC aged 18 or older who initiated 
prenatal care at a KPNC medical facility participating in the pregnancy cohort were invited to participate in the RPGEH pregnancy cohort. Among the 93,409 pregnancies occurring at medical facilities participating in the RPGEH pregnancy cohort during this initial recruitment period, 16,977 RPGEH pregnancy cohort consent forms were received, which represents a participation rate of $18.2 \%$. Compared to non-participants, women who participated in the pregnancy cohort were similar in age, but were more likely to have initiated prenatal care in the first trimester and to be non-Hispanic white and were slightly less likely to be Asian (Table 1). RPGEH pregnancy cohort participants were KPNC members for an average of 10 years before their pregnancy (Table 1). Among the 16,977 RPGEH pregnancy cohort participants who delivered a liveborn infant at the time of this writing, $93.2 \%$ had a first trimester blood draw (mean gestational age: 9.1 weeks +/-4.2 SD) and $80.5 \%$ had a second trimester blood draw (mean gestational age: 18.1 weeks $+/-5.5 \mathrm{SD}$ ) and $77.6 \%$ had blood drawn in both trimesters.

Of the 93,409 pregnancies initially identified, 80,086 (84 \%) delivered an infant in Kaiser Permanente Northern California. Of the pregnancies not resulting in livebirths, $5.7 \%$ were due to pregnancy loss, $4.6 \%$ no longer had Kaiser medical coverage, and $4.0 \%$ delivered outside of Kaiser. Among the deliveries in Kaiser Permanente Northern California, the prevalence of preterm birth ( $<37$ weeks), cesarean delivery, small for gestational age, large for gestational age, macrosomia, preeclampsia, GDM and NICU admissions was similar between RPGEH pregnancy cohort participants and non-participants (none of these outcomes differed by more than $1.2 \%$; see Table 2).

Participants were slightly more likely to be screened for GDM (95.6 \% versus $93.1 \%$ ). Overall, participants and non-participants were very similar in their behavioral risk factors assessed on the Early Start Questionnaire at the first prenatal visit (Table 3). Participants and non-participants did not differ in terms of smoking during the 12 months before pregnancy or during pregnancy. However, participants were slightly more likely to report drinking alcohol both before pregnancy (Table 3).

The use of RPGEH Pregnancy Cohort specimens and data are governed by the guiding principles of use and access established by the RPGEH. These principles include: 1) promote good science for the benefit of the public; 2) protect participant confidentiality and privacy; honor commitments made to participants and act within the scope of their consent; and preserve the trust that KPNC members have in KPNC; 3) comply with applicable legal and regulatory requirements; 4) consider whether the Resource is the best or only resource to address proposed research questions; 5) conserve limited
Table 1 Characteristics of RPGEH Prenatal Cohort Participants Compared to Non-Participants in Kaiser Permanente Northern California

\begin{tabular}{|c|c|c|c|}
\hline & $\begin{array}{l}\text { Overall } \\
\text { Denominator } \\
(n=93,409)\end{array}$ & $\begin{array}{l}\text { Participants } \\
(n=16,977)\end{array}$ & $\begin{array}{l}\text { Non-Participants } \\
(n=76,432)\end{array}$ \\
\hline Characteristic & N (\%) & & \\
\hline \multicolumn{4}{|l|}{$\mathrm{Age}^{\mathrm{a}}$} \\
\hline $18-24$ & $14,590(15.6)$ & $2,456(15.1)$ & $12,134(15.9)$ \\
\hline $25-29$ & $25,685(27.5)$ & $4,769(28.1)$ & $20,916(27.4)$ \\
\hline $30-34$ & $32,469(34.8)$ & $6,158(36.3)$ & $26,311(34.4)$ \\
\hline $35-39$ & $16,574(17.7)$ & $2,940(17.9)$ & $13,634(17.8)$ \\
\hline 40 or older & $4,065(4.4)$ & $653(3.8)$ & $3,412(4.5)$ \\
\hline Unknown & $26(<0.1)$ & $1(<0.1)$ & $25(<0.1)$ \\
\hline \multicolumn{4}{|l|}{ Race/Ethnicity } \\
\hline Non-Hispanic White & $36,704(39.3)$ & $7,975(47.0)$ & $28,729(37.6)$ \\
\hline Hispanic & $22,578(24.2)$ & $3,909(23.0)$ & $18,669(24.4)$ \\
\hline Asian & $22,413(24.0)$ & $3,264(19.2)$ & $19,149(25.0)$ \\
\hline African American & $6,448(6.9)$ & $960(5.6)$ & $5,488(7.2)$ \\
\hline Other & $3,965(4.2)$ & $691(4.1)$ & $3,274(4.3)$ \\
\hline Unknown & $1,301(1.4)$ & $178(1.1)$ & $1,123(1.5)$ \\
\hline \multicolumn{4}{|l|}{ Parity } \\
\hline 0 & $58,395(62.5)$ & $10,312(60.7)$ & $48,083(62.9)$ \\
\hline 1 & $26,210(28.1)$ & $5,030(29.6)$ & $21,180(27.7)$ \\
\hline $2+$ & $8,804(9.4)$ & $1,635(9.6)$ & $7,169(9.4)$ \\
\hline \multicolumn{4}{|l|}{ Pre-pregnancy BMI $\left(\mathrm{kg} / \mathrm{m}^{2}\right)$} \\
\hline$<18.5$ & $1919(2.4)$ & $276(1.9)$ & $1,643(2.5)$ \\
\hline $18.5-24.9$ & $36,957(46.2)$ & $6,607(44.8)$ & $30,350(46.5)$ \\
\hline $25.0-29.9$ & $20,758(25.9)$ & $4,013(27.2)$ & $16,745(25.6)$ \\
\hline $30.0+$ & $17,132(21.4)$ & $3546(24.0)$ & $13,586(20.8)$ \\
\hline Unknown & $3,320(4.2)$ & $315(2.1)$ & $3,005(4.6)$ \\
\hline \multicolumn{4}{|c|}{ Gestational age at initiation of prenatal care } \\
\hline 1st trimester & $83,551(89.5)$ & $16,041(94.5)$ & $67,510(88.3)$ \\
\hline 2nd trimester & $6,707(7.2)$ & $722(4.3)$ & $5,985(7.8)$ \\
\hline 3rd trimester & $2,980(3.2)$ & $141(0.8)$ & $2,839(3.7)$ \\
\hline Unknown & $171(0.2)$ & $73(0.4)$ & $98(0.1)$ \\
\hline $\begin{array}{l}\text { Length of enrollment } \\
\text { before pregnancy } \\
\text { (years) }\end{array}$ & $8.9(8.6)$ & $10.0(9.1)$ & $8.6(8.5)$ \\
\hline $\begin{array}{l}\text { Length of enrollment } \\
\text { after pregnancy (years) }\end{array}$ & $2.5(1.2)$ & $2.7(1.3)$ & $2.5(1.2)$ \\
\hline
\end{tabular}

${ }^{\mathrm{a} A g e}$ at standard first trimester prenatal panel blood draw

materials or resources for high-value research, such as biospecimens, which can be exhausted, and use of biospecimens that are rare or of higher value because of the data associated with them; 6) ensure that an investigator at the KPNC Division of Research (DOR) is involved in the research question and the conduct of the study to ensure the right and appropriate use of the 
Table 2 Perinatal Outcomes of the RPGEH Prenatal Cohort Participants Compared to Non-Participants in Kaiser Permanente Northern California

\begin{tabular}{|c|c|c|c|}
\hline & $\begin{array}{l}\text { Overall } \\
\text { Denominator } \\
(n=80,086)\end{array}$ & $\begin{array}{l}\text { Participants } \\
(n=14,757)\end{array}$ & $\begin{array}{l}\text { Non-Participant } \\
(n=65,329)\end{array}$ \\
\hline Perinatal Outcome & & N (\%) & \\
\hline \multicolumn{4}{|l|}{ Preeclampsia } \\
\hline None & $71,368(89.1)$ & $13,017(88.2)$ & $58,315(89.3)$ \\
\hline $\begin{array}{l}\text { Pre-existing } \\
\text { hypertension }\end{array}$ & $2,441(3.1)$ & $524(3.4)$ & $1,917(2.9)$ \\
\hline $\begin{array}{l}\text { Gestational } \\
\text { hypertension }\end{array}$ & 3,079 (3.8) & $604(4.1)$ & 2,475 (3.8) \\
\hline $\begin{array}{l}\text { Preeclampsia/ } \\
\text { Eclampsia }\end{array}$ & $3,198(4.0)$ & $612(4.1)$ & $2,586(4.0)$ \\
\hline \multicolumn{4}{|l|}{ Glucose Tolerance Status } \\
\hline Normal Screening & $56,461(70.5)$ & 10,861 (73.6) & $45,599(69.8)$ \\
\hline Abnormal Screening & $12,013(15.0)$ & $2,125(14.4)$ & $9,930(15.2)$ \\
\hline Gestational Diabetes & $5,366(6.7)$ & $959(6.5)$ & $4,508(6.9)$ \\
\hline Preexisting T2DM & $961(1.2)$ & $162(1.1)$ & $784(1.2)$ \\
\hline Not Screened & $4,805(6.4)$ & $649(4.4)$ & $4,508(6.9)$ \\
\hline \multicolumn{4}{|l|}{ Gestational Age } \\
\hline$<37$ weeks & $6,063(7.6)$ & $1,095(7.4)$ & $4,968(7.6)$ \\
\hline$\geq 37$ weeks & $74,005(92.4)$ & $13,662(92.6)$ & $60,343(92.4)$ \\
\hline Unknown & $18(<0.1)$ & $0(0)$ & $18(<0.1)$ \\
\hline \multicolumn{4}{|l|}{ Gestational Age } \\
\hline $\begin{array}{l}\text { Extreme Preterm } \\
(<28 \text { weeks })\end{array}$ & $324(0.4)$ & $57(0.4)$ & $267(0.4)$ \\
\hline $\begin{array}{l}\text { Severe Preterm } \\
\text { (28-31 week) }\end{array}$ & $526(0.7)$ & $91(0.6)$ & $435(0.7)$ \\
\hline $\begin{array}{l}\text { Moderate Preterm } \\
\text { (32-33 weeks) }\end{array}$ & $698(0.9)$ & $116(0.8)$ & $582(0.9)$ \\
\hline $\begin{array}{l}\text { Late Preterm } \\
\text { (34-36 weeks) }\end{array}$ & $4,515(5.6)$ & $831(5.6)$ & $3,684(5.6)$ \\
\hline$\geq 37$ weeks & $74,005(92.4)$ & $13,662(92.6)$ & 60,343 (92.4) \\
\hline Unknown & $18(<0.1)$ & $0(0)$ & $18(<0.1)$ \\
\hline
\end{tabular}

Gestational weight gain in relation to the IOM weight gain guidelines

\begin{tabular}{|c|c|c|c|}
\hline Below & $10,456(13.1)$ & $1,847(12.5)$ & 8,609 (13.2) \\
\hline Met & $17,196(21.5)$ & 3,115 (21.1) & 14,081 (21.6) \\
\hline Exceeded & $48,689(60.8)$ & $9,416(63.8)$ & $39,273(60.1)$ \\
\hline Unknown & $3,745(4.7)$ & 379 (2.6) & $3,366(5.2)$ \\
\hline $\begin{array}{l}\text { Infant birth weight } \\
\text { (grams) }^{a}\end{array}$ & $3,361 \pm 556$ & $3,400 \pm 565$ & $3,350 \pm 553$ \\
\hline AGA & $63,843(81.4)$ & $11,771(81.3)$ & $52,072(81.4)$ \\
\hline SGA & $7,436(9.5)$ & $1,339(9.3)$ & $6,097(9.5)$ \\
\hline LGA & $6,992(8.9)$ & $1,342(9.3)$ & $5,650(8.8)$ \\
\hline Unknown & $135(0.2)$ & $17(0.1)$ & $118(0.2)$ \\
\hline
\end{tabular}

Infant birth weight (grams)

Very low birth weight $605(0.8) \quad 106(0.7) \quad 499(0.8)$ $(<1,500)$
Table 2 Perinatal Outcomes of the RPGEH Prenatal Cohort Participants Compared to Non-Participants in Kaiser Permanente Northern California (Continued)

\begin{tabular}{llll}
\hline $\begin{array}{l}\text { Low birth weight } \\
(1,500-2,500)\end{array}$ & $3,158(4.0)$ & $555(3.8)$ & $2,603(4.1)$ \\
$\begin{array}{l}\text { Normal birth weight } \\
(2,501-3,999)\end{array}$ & $65,975(84.2)$ & $12,042(83.2)$ & $53,933(84.4)$ \\
Macrosomia (4,000+) & $8,582(10.9)$ & $1,757(12.2)$ & $6,825(10.7)$ \\
Unknown & $86(0.1)$ & $9(0.1)$ & $77(0.1)$ \\
NICU Stay & & & \\
Non-NICU & $71,563(89.4)$ & $13,092(88.7)$ & $58,471(89.5)$ \\
NICU & $8,393(10.5)$ & $1,647(11.2)$ & $6,746(10.3)$ \\
Delivery room death & $112(0.1)$ & $17(0.1)$ & $95(0.2)$ \\
Unknown & $18(<0.1)$ & $1(<0.1)$ & $17(<0.1)$ \\
\hline
\end{tabular}

aGA is large-for-gestational age, adjusted for race/ethnicity

SGA is small-for-gestational age, adjusted for race/ethnicity

AGA is appropriate for-gestational age, adjusted for race/ethnicity

resources. Applications for use of RPGEH Pregnancy Cohort samples and data are submitted and reviewed by the RPGEH Access Review Committee (ARC). The ARC meets three times a year to review applications for use of RPGEH data and specimens. The ARC includes DOR investigators, plus external stakeholders and investigators to address specific content and methodological issues as required by the projects under consideration. The ARC governs access to and use of all RPGEH data and specimens by requestors. Applications for access will be subject to three phases of review, and the ARC's decisions are made based on a formalized set of criteria that can be reviewed.

Statistical analyses, power and sample size considerations Based on our expected cohort size of 25,000 women we computed power for hypothetical case-control studies. We assumed all available cases will be included and controls will be sampled at a ratio of 5:1. We computed the minimum detectable odds ratio (OR) for a two-sided test at level 0.05 and $80 \%$ power for several outcomes with different prevalences. For the outcome of small for gestational age (prevalence $9.3 \%$ ) a case-control study will be powered to detect an OR of 1.15. For the outcome of gestational hypertension (prevalence $4.1 \%$ ) a casecontrol study will be powered to detect an OR of 1.22. For the rare outcome of very low birthweight (prevalence $0.7 \%$ ) a case-control study will be powered to detect an OR of 1.57 .

\section{Discussion}

This report provides a brief overview of the establishment of the KPNC RPGEH Pregnancy Cohort and its biorepository, which were created to provide a resource 
Table 3 Substance use before and during pregnancy among RPGEH Prenatal Cohort Participants compared to NonParticipants in Kaiser Permanente Northern California

\begin{tabular}{|c|c|c|c|}
\hline & $\begin{array}{l}\text { Overall } \\
\text { Denominator } \\
(n=87,928)\end{array}$ & $\begin{array}{l}\text { Participants } \\
(n=16,584)\end{array}$ & $\begin{array}{l}\text { Non-Participan } \\
(n=71,344)\end{array}$ \\
\hline Early Start Data & & N (\%) & \\
\hline \multicolumn{4}{|c|}{ Smoking in 12 Months before Pregnancy } \\
\hline Never & $78,710(89.5)$ & $14,683(88.5)$ & $64,027(89.7)$ \\
\hline Monthly or Less & $2,944(3.4)$ & $623(3.8)$ & $2,321(3.3)$ \\
\hline Weekly & $1,304(1.5)$ & $230(1.4)$ & $1,074(1.5)$ \\
\hline Daily & $4,747(5.4)$ & $1,016(6.1)$ & $3,731(5.2)$ \\
\hline Unknown & $223(0.2)$ & $32(0.2)$ & $191(0.3)$ \\
\hline \multicolumn{4}{|c|}{ Smoking since Pregnancy } \\
\hline Never & $85,166(96.9)$ & $16,010(96.5)$ & $69,156(96.9)$ \\
\hline Monthly or Less & $789(0.9)$ & $159(1.0)$ & $630(0.9)$ \\
\hline Weekly & $537(0.6)$ & $117(0.7)$ & $420(0.6)$ \\
\hline Daily & $1,196(1.3)$ & $267(1.6)$ & $929(1.3)$ \\
\hline Unknown & $240(0.3)$ & $31(0.2)$ & $209(0.3)$ \\
\hline \multicolumn{4}{|c|}{ Alcohol in 12 Months before Pregnancy } \\
\hline Never & $28,267(32.2)$ & $4,202(25.3)$ & $24,065(33.7)$ \\
\hline Monthly or less & $39,589(45.0)$ & $7,908(47.7)$ & $31,681(44.4)$ \\
\hline Weekly & $18,102(20.6)$ & $4,058(24.5)$ & $14,044(19.7)$ \\
\hline Daily & $1,429(1.6)$ & $327(2.0)$ & $1,102(1.6)$ \\
\hline Unknown & $541(0.6)$ & $89(0.5)$ & $452(0.6)$ \\
\hline \multicolumn{4}{|c|}{ Alcohol since Pregnancy } \\
\hline Never & $78,796(89.6)$ & $14,551(87.7)$ & $64,245(90.1)$ \\
\hline Monthly or Less & $6,780(7.7)$ & $1,517(9.2)$ & $5,263(7.3)$ \\
\hline Weekly & $1,423(1.6)$ & $344(2.1)$ & $1,079(1.5)$ \\
\hline Daily & $179(0.2)$ & $34(0.2)$ & $145(0.2)$ \\
\hline Unknown & 750 (0.9) & $138(0.8)$ & $612(0.9)$ \\
\hline
\end{tabular}

Use of Prescription Drug in 12 Months before Pregnancy ${ }^{\mathrm{a}}$

$\begin{array}{llll}\text { No } & 71,212(81.0) & 13,067(78.8) & 58,145(81.5) \\ \text { Yes } & 16,066(18.3) & 3,403(20.5) & 12,663(17.7) \\ \text { Unknown } & 650(0.7) & 114(0.7) & 536(0.8)\end{array}$

Use of Prescription Drug since Pregnancy

$\begin{array}{llll}\text { No } & 83,680(95.2) & 15,726(94.8) & 67,954(95.3) \\ \text { Yes } & 3,871(4.4) & 799(4.8) & 3,072(4.3) \\ \text { Unknown } & 377(0.4) & 59(0.4) & 318(0.4)\end{array}$

Use of Illegal Drug in 12 Months before Pregnancy ${ }^{\mathrm{b}}$

$\begin{array}{llll}\text { No } & 79,954(90.9) & 14,790(89.1) & 65,164(91.3) \\ \text { Yes } & 7,149(8.1) & 1,506(9.1) & 5,643(7.9) \\ \text { Unknown } & 825(1.0) & 288(1.8) & 537(0.8)\end{array}$

Table 3 Substance use before and during pregnancy among RPGEH Prenatal Cohort Participants compared to NonParticipants in Kaiser Permanente Northern California (Continued)

\begin{tabular}{llll}
\hline Use of Illegal Drug since Pregnancy & & \\
No & $85,202(96.9)$ & $15,878(95.7)$ & $69,324(97.2)$ \\
Yes & $2,033(2.3)$ & $414(2.5)$ & $1,619(2.3)$ \\
Unknown & $693(0.8)$ & $292(1.8)$ & $401(0.5)$
\end{tabular}

Prescription drugs in Early Start data include anxiety medications, pain medications, and sleep medications

blllegal drugs in Early Start data include Cocaine, Heroin, Marijuana, and Methamphetamines

for women's and children's health research. The KPNC RPGEH Pregnancy Cohort is uniquely integrated into routine clinical prenatal care within the KPNC health care system setting and can be linked with data from the EHR. KPNC contains a racially and ethnically diverse population, thereby increasing the likelihood of obtaining a highly representative sample with generalizable findings.

The establishment of this valuable resource has the potential to address many key questions related to women's and children's health and is particularly timely, in light of the recent dissolution of The National Children's Study. The National Children's Study (NCS) was developed after a 1990s White House Task Force highlighted the paucity of evidence evaluating the links between environmental exposures, development, and health outcomes in children and adults. The Children's Health Act of 2000 initiated the conduct of a national longitudinal study of environmental influences (including physical, chemical, biological, and psychosocial) during pregnancy on child health and development. A recent report explains that this study was dissolved due to feasibility and oversight issues [21, 22] and suggests that funding agencies support smaller focused studies designed as tailored explorations as well as cohorts to facilitate longitudinal biospecimen collection and banking.

This large pregnancy cohort, derived from a diverse base population, can be used to generate sets of cases and controls for future clinical research studies, as demonstrated by our preliminary data. The availability of rich clinical data from the EHR, the questionnaire data, and existing perinatal research programs provide detailed phenotypic information that will further facilitate the conduct of perinatal epidemiology and translational studies. The RPGEH Pregnancy Cohort, coupled with the state of the art KPNC Biorepository for long-term storage of serum, plasma and DNA samples and an ability to follow both women and their child long term for future health outcomes in the EHR, provides a truly unique and valuable resource for improving our understanding of women and children's health. 
Our preliminary data on the RPGEH Pregnancy Cohort demonstrate that at least $18.2 \%$ of pregnant women participated, and the cohort is highly representative of the underlying KPNC pregnant population in terms of both maternal demographics and key perinatal outcomes. Pregnancy cohort participants were KNPC members on average 10 years before their index pregnancy and remained members on average 2.7 years after pregnancy to date, and most are still currently KPNC members. Thus, there is a unique ability to examine exposures even years before pregnancy and to follow women and their infants for years after delivery. While participating women were slightly more likely to be non-Hispanic white and less likely to be Asian, this pattern is frequently observed in cohort studies with multiethnic populations such as KPNC women of reproductive age. Overall, the RPGEH Pregnancy Cohort is extremely diverse, with $53 \%$ of participants from non-white racial ethnic minority groups, and Asian women comprise $23 \%$ of the cohort. This is especially significant as Asian women have previously been reported as less likely to participate in reproductive and biospecimen research [22, 23]. The racial-ethnic diversity of this population provides important potential for studies examining racial-ethnic disparities in diseases and health care delivery. Given the recruitment efforts integration within clinical care, it is possible that not all pregnant women at participating medical facilities were invited to participate in the pregnancy cohort; therefore, $18.2 \%$ is likely an underestimate of the overall participation rate.

The prevalence of several perinatal complications was similar between RPGEH cohort participants and the underlying populations of women delivering in KPNC. Cohort participants were slightly less likely to have gestational diabetes mellitus (GDM) and infants of participants were slightly more likely to be macrosomic relative to non-participants. The lower prevalence of GDM among RPGEH participants is probably due in part to the fact that participants were less likely to be Asian and more likely to be non-Hispanic white; in this setting, Asian woman have the highest prevalence of $\operatorname{GDM}[15,24]$ and non-Hispanic white women have the lowest prevalence of GDM.

The fetal origins of adult disease hypothesis posits that "fetal programming" occurs when maternal metabolic nutrition, environment and hormonal milieu during development permanently programs the structure and physiology of organs and hence the future health of the offspring [25]. While there is some epidemiologic evidence supporting the "fetal programming" hypothesis, more longitudinal, observational studies examining the effects of a broad range of environmental and biological factors assessed in utero are needed to clarify the extent to which fetal programming contributes to adult diseases. In addition, a woman's health status during pregnancy may also influence her future health [26]. For example, women diagnosed with pregnancy-related hypertension and/or preeclampsia, gestational diabetes and preterm birth are at higher risk for hypertension, diabetes and cardiovascular disease later in life [7]. Therefore, given the rich health data in the KPNC EHR, the RPGEH Pregnancy Cohort will also allow for a lifecourse research approach [27].

The resource is available to be used by Kaiser Permanente researchers as well as outside investigators who wish to collaborate with a Kaiser Permanente researcher to conduct biomarker, genetic, environmental and gene environment interaction studies. The RPGEH Pregnancy Cohort has the unique ability to connect biospecimens collected at two time points during pregnancy with detailed short- and long-term environmental and clinical data on both women and their children, enabling research of immediate perinatal complications as well as longer term maternal, child, and adult outcomes.

\section{Additional file}

Additional file 1: Appendix 1. PG En Survey 2012-02-17 Pregnancy Cohort survey. (DOCX $33 \mathrm{~kb}$ )

Abbreviations

ARC: Access review committee; DOR: Division of Research; EDTA: Ethylenediaminetetraacetic acid; EHR: Electronic health record; GDM: Gestational diabetes mellitus; KPNC: Kaiser Permanente Northern California; LMP: Last menstrual period; NCS: National children's study; RPGEH: Research Program on Genes Environment and Health; SST: Serum separator tube

\section{Acknowledgements}

This work was supported by grant RC2 AG036607 from the National Institutes of Health, grants from the Robert Wood Johnson Foundation, and Kaiser Permanente Northern California Community Benefit. We are grateful to the Kaiser Permanente Northern California Members who have generously agreed to participate in the Research Program on Genes, Environment and Health.

\section{Funding}

This work was supported by grant RC2 AG036607 from the National Institutes of Health, grants from the Robert Wood Johnson Foundation, and Kaiser Permanente Northern California Community Benefit. Drs. Hedderson, Avalos, Ferrara and Croen received support from UG3OD0 23289 for this work.

\section{Availability of data and material}

The datasets generated during and/or analysed during the current study are not publicly available due to the fact that the data used for this study contain protected health information (PHI). Kaiser Permanente IRB policies prohibit releasing PHI. Data are available from the Kaiser Permanente Division of Research for researchers who meet the criteria for access to confidential data from the corresponding author on reasonable request.

\section{Authors' contributions}

$\mathrm{MMH}$. Overseeing the data analysis, interpretation of the data and drafting the manuscript and revising it critically for important intellectual content. AF. Drafting the manuscript and revising it critically for important intellectual content. LAA. Drafting the manuscript and revising it critically for important intellectual content. SKV. Made significant contributions to the design and 
drafting the manuscript. EPG. Drafting the manuscript and revising it critically for important intellectual content. DKL. Drafting the manuscript and revising it critically for important intellectual content. AA. Involved in acquisition of the data and drafting the manuscript. SW Involved in acquisition of the data and drafting the manuscript. SR Involved in acquisition of the data and drafting the manuscript. CS. Made significant contributions to the conception and design and drafting the manuscript. LAC. made significant contributions to the design and drafting the manuscript. TF. Drafting the manuscript and revising it critically for important intellectual content. FX. Acquisition of data and data analysis. VC. Acquisition of data and data analysis. All read and approved the final manuscript.

\section{Competing interests}

The authors declare that they have no competing interests.

\section{Consent for publication}

Not applicable.

\section{Ethics approval and consent to participate}

We obtained ethics approval and consent from the human subjects committee of the Kaiser Foundation Research Institute; the project reference number is CN-05CScha-04-H. Ethical approval covers all sites included in the study. All study participants provided written informed consent and all data assessment tools and components for the RPGEH pregnancy cohort have been approved by the human subjects committee of the Kaiser Foundation Research Institute.

Received: 16 January 2016 Accepted: 8 November 2016

Published online: 29 November 2016

\section{References}

1. Dabelea D, Crume T. Maternal environment and the transgenerational cycle of obesity and diabetes. Diabetes. 2011;60:1849-55.

2. Gluckman PD, Hanson MA, Cooper C, Thornburg KL. Effect of in utero and early-life conditions on adult health and disease. N Engl J Med. 2008;359:61-73.

3. Ben-Shlomo Y, Kuh D. A life course approach to chronic disease epidemiology: conceptual models, empirical challenges and interdisciplinary perspectives. Int J Epidemiol. 2002;31:285-93. 11980781.

4. Bellamy L, Casas JP, Hingorani AD, Williams DJ. Pre-eclampsia and risk of cardiovascular disease and cancer in later life: systematic review and metaanalysis. BMJ. 2007;335:974.

5. Bellamy L, Casas JP, Hingorani AD, Williams D. Type 2 diabetes mellitus after gestational diabetes: a systematic review and meta-analysis. Lancet. 2009;373:1773-9.

6. Catov JM, Newman AB, Roberts JM, et al. Preterm delivery and later maternal cardiovascular disease risk. Epidemiology. 2007;18:733-9.

7. Fraser A, Nelson SM, Macdonald-Wallis C, et al. Associations of pregnancy complications with calculated cardiovascular disease risk and cardiovascular risk factors in middle age: the Avon Longitudinal Study of Parents and Children. Circulation. 2012;125:1367-80.

8. Krieger N. Overcoming the absence of socioeconomic data in medical records: validation and application of a census-based methodology. Am J Public Health. 1992;82:703-10.

9. Spitzer RL, Williams JB, Kroenke K, Hornyak R, McMurray J. Validity and utility of the PRIME-MD patient health questionnaire in assessment of 3000 obstetric-gynecologic patients: the PRIME-MD Patient Health Questionnaire Obstetrics-Gynecology Study. Am J Obstet Gynecol. 2000;183:759-69.

10. Spitzer RL, Kroenke K, Williams JB. Validation and utility of a self-report version of PRIME-MD: the PHQ primary care study. Primary Care Evaluation of Mental Disorders. Patient Health Questionnaire. JAMA. 1999;282:1737-44.

11. Kroenke K, Spitzer RL, Williams JB, Monahan PO, Lowe B. Anxiety disorders in primary care: prevalence, impairment, comorbidity, and detection. Ann Intern Med. 2007;146:317-25. 17339617.

12. Armstrong MA, Lieberman L, Carpenter DM, et al. Early Start: an obstetric clinic-based, perinatal substance abuse intervention program. Qual Manag Health Care. 2001;9:6-15.

13. Weight Gain During Pregnancy. Reexamining the Guidelines. Washingtion: National Academies Press; 2009.

14. Selby JV, Newman B, King MC, Friedman GD. Environmental and behavioral determinants of fasting plasma glucose in women. A matched co-twin analysis. Am J Epidemiol. 1987;125:979-88.
15. Ferrara A, Kahn HS, Quesenberry C, Riley C, Hedderson MM. An increase in the incidence of gestational diabetes mellitus: Northern California, 1991-2000. Obstet Gynecol. 2004;103:526-33.

16. Committee opinion no. 504: screening and diagnosis of gestational diabetes mellitus. Obstet Gynecol. 2011;118:751-3.

17. Hedderson MM, Darbinian JA, Sridhar SB, Quesenberry CP. Prepregnancy cardiometabolic and inflammatory risk factors and subsequent risk of hypertensive disorders of pregnancy. Am J Obstet Gynecol. 2012;207:68-9. 22727352.

18. Goldenberg RL, Culhane JF, lams JD, Romero R. Epidemiology and causes of preterm birth. Lancet. 2008;371:75-84. 18177778.

19. Ehrlich SF, Crites YM, Hedderson MM, Darbinian JA, Ferrara A. The risk of large for gestational age across increasing categories of pregnancy glycemia. Am J Obstet Gynecol. 2011;204.

20. Escobar GJ, Fischer A, Kremers R, Usatin MS, Macedo AM, Gardner MN Rapid retrieval of neonatal outcomes data: the Kaiser Permanente Neonatal Minimum Data Set. Qual Manag Health Care. 1997:5:19-33.

21. National Institutes of Health. National Children's Study (NCS) Working Group: Final Report - December 12, 2014. 2014.

22. Talaulikar VS, Hussain S, Perera A, Manyonda IT. Low participation rates amongst Asian women: implications for research in reproductive medicine. Eur J Obstet Gynecol Reprod Biol. 2014;174:1-4.

23. Lee $\mathrm{Cl}$, Bassett $\mathrm{LW}$, Leng $\mathrm{M}$, et al. Patients' willingness to participate in a breast cancer biobank at screening mammogram. Breast Cancer Res Treat 2012;136:899-906

24. Hedderson M, Ehrlich S, Sridhar S, Darbinian J, Moore S, Ferrara A. Racial/ ethnic disparities in the prevalence of gestational diabetes mellitus by BMI. Diabetes Care. 2012;35:1492-8.

25. Barker DJ. The origins of the developmental origins theory. J Intern Med. 2007:261:412-7. 17444880.

26. Saade GR. Pregnancy as a window to future health. Obstet Gynecol. 2009; 114:958-60. 20168094.

27. Callahan T, Stampfel C, Cornell A, et al. From Theory to Measurement: Recommended State MCH Life Course Indicators. Matern Child Health J. 2015:19:2336-47. 26122251.

\section{Submit your next manuscript to BioMed Central and we will help you at every step:}

- We accept pre-submission inquiries

- Our selector tool helps you to find the most relevant journal

- We provide round the clock customer support

- Convenient online submission

- Thorough peer review

- Inclusion in PubMed and all major indexing services

- Maximum visibility for your research

Submit your manuscript at www.biomedcentral.com/submit
) BioMed Central 